\title{
Single-cell analysis of mouse uterus at the invasion phase of embryo implantation
}

\author{
Jia-Peng He, Qing Tian, Qiu-Yang Zhu and Ji-Long Liu* (D)
}

\begin{abstract}
Background: Embryo implantation into the uterus is a crucial step for human reproduction. A hypothesis has been proposed that the molecular circuit invented by trophoblasts for invasive embryo implantation during evolution might be misused by cancer cells to promote malignancy. Unfortunately, our current understanding of the molecular mechanism underlying embryo implantation is far from complete.

Results: Here we used the mouse as an animal model and generated a single-cell transcriptomic atlas of the embryo implantation site of mouse uterus at the invasion phase of embryo implantation on gestational day 6 . We revealed 23 distinct cell clusters, including 5 stromal cell clusters, 2 epithelial cell clusters, 1 smooth muscle cell cluster, 2 pericyte clusters, 4 endothelial cell clusters, and 9 immune cell clusters. Through data analysis, we identified differentially expression changes in all uterine cell types upon embryo implantation. By integrated with single-cell RNA-seq data from E5.5 embryos, we predicted cell-cell crosstalk between trophoblasts and uterine cell types.
\end{abstract}

Conclusions: Our study provides a valuable resource for understanding of the molecular mechanism of embryo implantation.

Keywords: Embryo implantation, Mouse, Single-cell RNA-seq, Transcriptional changes

\section{Background}

Embryo implantation in humans is interstitial. It consists of the following three phases: embryo apposition, attachment, and invasion. Immediately after apposition and attachment to endometrial epithelium, the blastocyst penetrates through the epithelium, followed by the basal lamina, and invades into the stroma [1]. It has been well established that excessive trophoblast invasion may lead to the pathogenesis of placenta creta [2], while shallow trophoblast invasion may result in pre-eclampsia and intrauterine growth restriction [3]. Interestingly, it has long been recognized that there are striking similarities between trophoblast cells and invasive cancer cells, both of which share proliferative, migratory and invasive

*Correspondence: jilongliu@scau.edu.cn

Guangdong Laboratory for Lingnan Modern Agriculture, College of Veterinary Medicine, South China Agricultural University, Tianhe District, No. 483 Wushan Road, Guangzhou 510642, China properties, as well as the capacity to confer immune privilege [4]. A hypothesis has been proposed that the molecular circuit invented by trophoblasts for invasive embryo implantation during evolution might be misused by cancer cells to promote malignancy [5]. Unfortunately, our current understanding of the mechanisms underlying embryo implantation is far from complete.

Due to ethical restrictions and experimental difficulties, in vivo analysis of embryo implantation heavily relies on mice [6]. Slightly different from humans, embryo implantation in mice is eccentric. The apposition phase of mouse embryo implantation is thought to occur from the morning to the midnight of gestational day 4 (GD4). During this phase, the embryo seeks its position on the luminal epithelium of uterus. After that, the embryo attaches to the receptive uterine epithelium. A firm connection between the blastocyst and uterine luminal epithelium is established on the morning of GD5. The invasion phase of embryo implantation occurs on GD6, when the original author(s) and the source, provide a link to the Creative Commons licence, and indicate if changes were made. The images or other third party material in this article are included in the article's Creative Commons licence, unless indicated otherwise in a credit line to the material. If material is not included in the article's Creative Commons licence and your intended use is not permitted by statutory regulation or exceeds the permitted use, you will need to obtain permission directly from the copyright holder. To view a copy of this licence, visit http://creativecommons.org/licenses/by/4.0/. The Creative Commons Public Domain Dedication waiver (http://creativecommons.org/publicdomain/zero/1.0/) applies to the data made available in this article, unless otherwise stated in a credit line to the data. 
blastocyst penetrates through the epithelium and invades into the stroma [7, 8]. Previously, two studies have analyzed global gene expression changes associated with the invasion phase of embryo implantation in mice by using high-throughput transcriptomic approaches $[9,10]$. The limitation of both studies was that the whole uterus was used. The uterus is a complex structure consisting of many cell types, including luminal and glandular epithelial cells, stromal cells, smooth muscle cells, endothelial cells, and various immune cells [11]. Thus, whole uterus transcriptomic studies were unable to accurately capture cell-type-specific gene expression changes.

In the present study, by using the-state-of-the-art single-cell RNA-seq approach [12], we resolved all cell types at the embryo implantation site of the mouse uterus on GD6. Through data analysis, we identified differentially expression changes in all cell types at the invasion phase of embryo implantation. We also predicted cell-cell crosstalk between trophoblasts and uterine cell types. Our study provides a valuable resource for understanding of the molecular mechanism of embryo implantation.

\section{Materials and methods Sample collection}

Adult CD-1 mice of the SPF grade were used in this study. All mice were caged under light-controlled conditions (14 h/10 h light/dark cycles) with free access to regular food and water. Female mice were mated with fertile males and success of mating was confirmed the next morning by the presence of a vaginal plug. The day of the vaginal plug was denoted as gestation day 1 (GD1). On GD6, the implantation sites and inter-implantation sites (served as a control) were collected separately. All animal procedures were approved by the Institutional Animal Care and Use Committee of South China Agricultural University (No. 2020B078, approved on 29/09/2020).

\section{Bulk RNA-seq analysis}

The total RNAs from uterine tissues were extracted with the TRIzol reagent (Invitrogen). RNA-seq libraries were generated by using the TruSeq RNA sample preparation kit (Illumina) and sequenced on an Illumina HiSeq 2500 system. Using UCSC mm10 mouse genome as reference, raw data were analyzed using TopHat v2.0.4 [13] and Cufflinks v2.2.1 [14] as described previously [15]. Gene expression levels were measured as fragments per kilobase per million (FPKM).

\section{Single-cell dissociation of mouse uterus}

Single-cell suspension was prepared as described previously $[16,17]$. Briefly, the uterine tissues from 3 mice for each group were pooled and minced with a blade. Tissues were then incubated in dissociation buffer containing
$2 \mathrm{mg} / \mathrm{ml}$ Collagenase II (\#C6885, Sigma-Aldrich), $10 \mathrm{mg} /$ $\mathrm{ml}$ Dispase II (\#354,235, Corning) and 50,000 U/ml DNase I (\#DN25, Sigma-Aldrich) for up to $30 \mathrm{~min}$ at $37^{\circ} \mathrm{C}$ in a shaking incubator. The digestion progress was checked every 5 min with a microscope until a single cell suspension was achieved. The single-cell suspension was then passed through a $40-\mu \mathrm{m}$ cell strainer to remove undigested tissues. Cells were spun down at $250 \mathrm{~g}$ at $4{ }^{\circ} \mathrm{C}$ for $4 \mathrm{~min}$ and the pelleted cells were washed using centrifugation. In order to measure cell viability, cells were strained with AO/PI solution (\#CS2-0106, Nexcelom Bioscience) and counted using a Cellometer Auto 2000 instrument (\#SD-100, Nexcelom Bioscience). The singlecell suspension was carried forward to single-cell RNAseq only if the cell viability was $>80 \%$ and the percentage of cell clumps was $<10 \%$.

\section{Single-cell RNA-seq library preparation and sequencing}

The final concentration of single-cell suspension was adjusted to 1000 cells $/ \mu \mathrm{l}$ and a volume of $15 \mu \mathrm{l}$ was loaded into one channel of the ChromiumTM Single Cell B Chip (\#1,000,073, $10 \times$ Genomics), aiming at recovering 8000-10,000 cells. The Chromium Single Cell 3' Library \& Gel Bead Kit v3 (\#1,000,075, $10 \times$ Genomics) was used for single-cell bar-coding, cDNA synthesis and library preparation, following the manufacturer's instructions provided as the Single Cell 3' Reagent Kits User Guide Version 3. Library sequencing was performed on an Illumina novaseq 6000 system configured with the paired-end 150-bp protocol at a sequencing depth of approximately 400 million reads.

\section{Single cell RNA-seq data analysis}

Raw data bcl files from the Illumina NovaSeq 6000 platform were converted to fastq files using the bcl2fastq tool (v2.19.0.316). These fastq files were aligned to the mm10 mouse reference genome by using the CellRanger software (v3.0.1, $10 \times$ Genomics). The resulting gene counts matrix was analyzed with the $R$ package Seurat (v3.1.3) [18]. Cell with fewer than 200 or greater than 6000 unique genes, as well as cells with greater than $25 \%$ of mitochondrial counts, were excluded. Meanwhile, genes expressed in fewer than 3 cells were removed. Following data filtering, the gene counts matrix was normalized and scaled by using NormalizeData and ScaleData, respectively. The top 2000 highest variable genes were used for the principal component analysis (PCA) and the optimal number of PCA components was determined by the JackStraw procedure. Single cells were clustered by the K-nearest neighbor (KNN) graph algorithm in PCA space and visualized using the $\mathrm{t}$-distributed stochastic neighbor embedding (tSNE) dimensional reduction technique. The cell type 
label for each cell cluster was manually assigned based on canonical cell markers. The FindAllMarkers function was used to identify novel marker genes for each cluster with a minimum of $10 \%$ of cells expressing the gene within the cluster and a minimum $\operatorname{logFC}$ threshold of 0.25. In order to find differential expressed genes in the same cell type between pre-receptive uterus and receptive uterus, the FindMarkers function in Seurat was used with min.logfc being set to 0.25 and min.pct being set to 0.20 .

\section{Pseudotime analysis}

Monocle2 package v2.18.0 [19] was used for pseudotime analysis. The count data and meta data were export from the Seurat object and then import into the CellDataSet object in Monocle2. Feature genes were selected by using the differentialGeneTest function. After The dimension reduction by using the DDRTree algorithm, the orderCells function was used to infer the trajectory with default parameters. The reconstructed trajectory was visualized by the plot_cell_trajectory function.

\section{Gene ontology analysis}

Gene ontology (GO) analysis was performed as described previously [20]. GO terms were grouped according to the biological process category in the Mouse Genome Informatics (MGI) GOslim database [21]. To test for enrichment, a hypergeometric test was conducted and $\mathrm{P}<0.05$ was used as significance threshold to identify enriched GO terms.

\section{Pathway analysis}

Pathway enrichment analysis was conducted by using the Metascape v7.4 online tools [22]. The significance threshold for FDR was set at 0.05 .

\section{Cell-cell communication analysis}

The CellChat v1.1.0 software [23] was used to infer cellcell communication based on ligand-receptor interaction with default parameters. For each ligand-receptor pair, CellChat assigned a communication probability value by the law of mass action based on the average expression values of a ligand by one cell group and that of a receptor by another cell group. The statistical significance of communication probability values was assessed by a permutation test. $\mathrm{P}<0.05$ were considered statistically significant.

\section{Results}

\section{A single-cell atlas of mouse uterus on gestational day 6}

To create a cell-type resolved map of mouse uterus at the invasion phase of embryo implantation, we performed single-cell RNA-seq analysis (Fig. 1A). The implantation site (IS) and the inter-implantation site (IIS) of mouse uterus were collected on gestational day 6 (GD6). The whole uterus, which is consist of endometrium, myometrium and perimetrium, was used. The embryo at IS was also kept. Single-cell RNA-seq data were generated by using the $10 \times$ Genomics platform. After quality control, a total of 16,257 cells (7065 for IIS and 9192 for IS) were

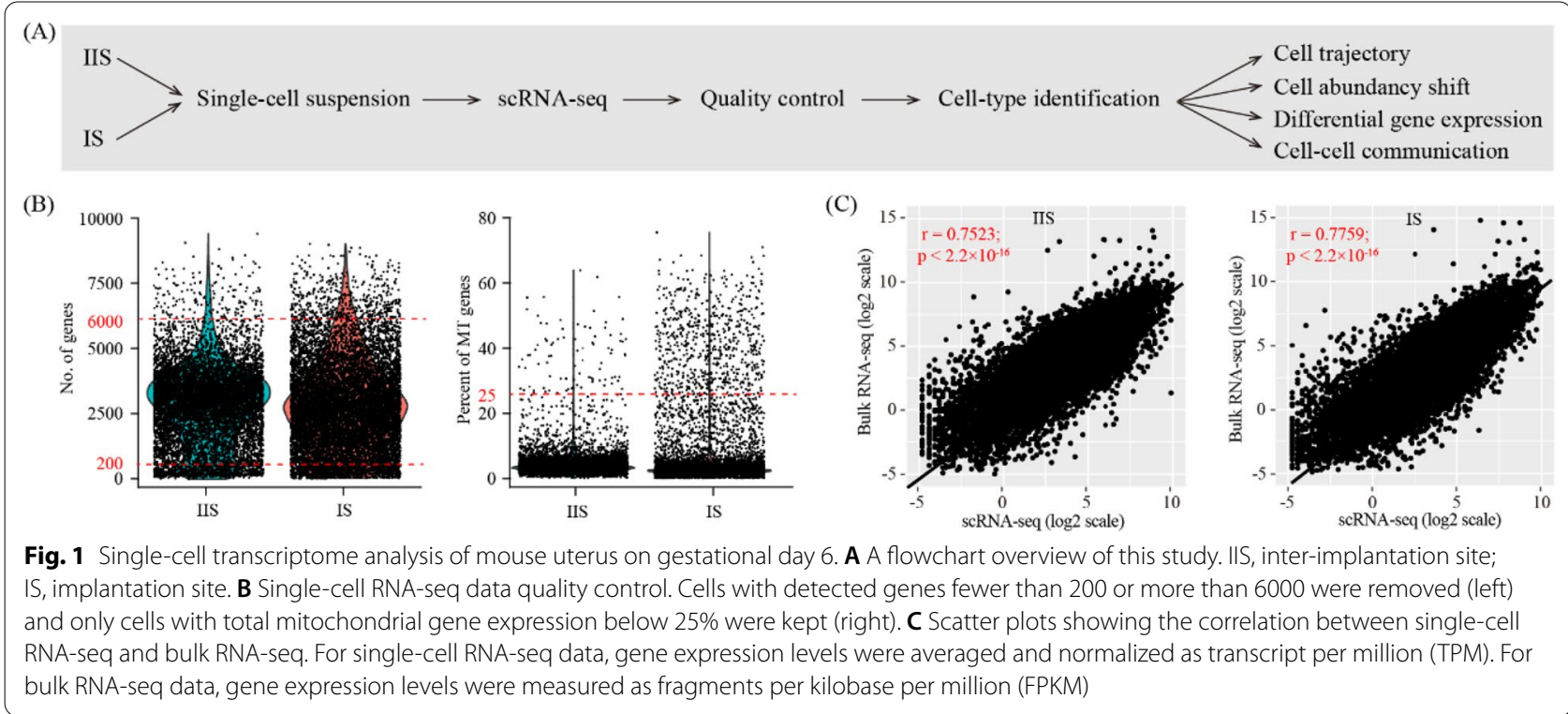


obtained (Fig. 1B). In order to validate this single-cell RNA-seq dataset, we also generate a bulk RNA-seq dataset using the same samples. It turned out that the cellaveraged single-cell RNA-seq data were highly accordant with the conventional bulk RNA-seq data $(r=0.7523$ for IIS and $r=0.7759$ for IS), indicative of high quality of our single-cell RNA-seq data (Fig. 1C).

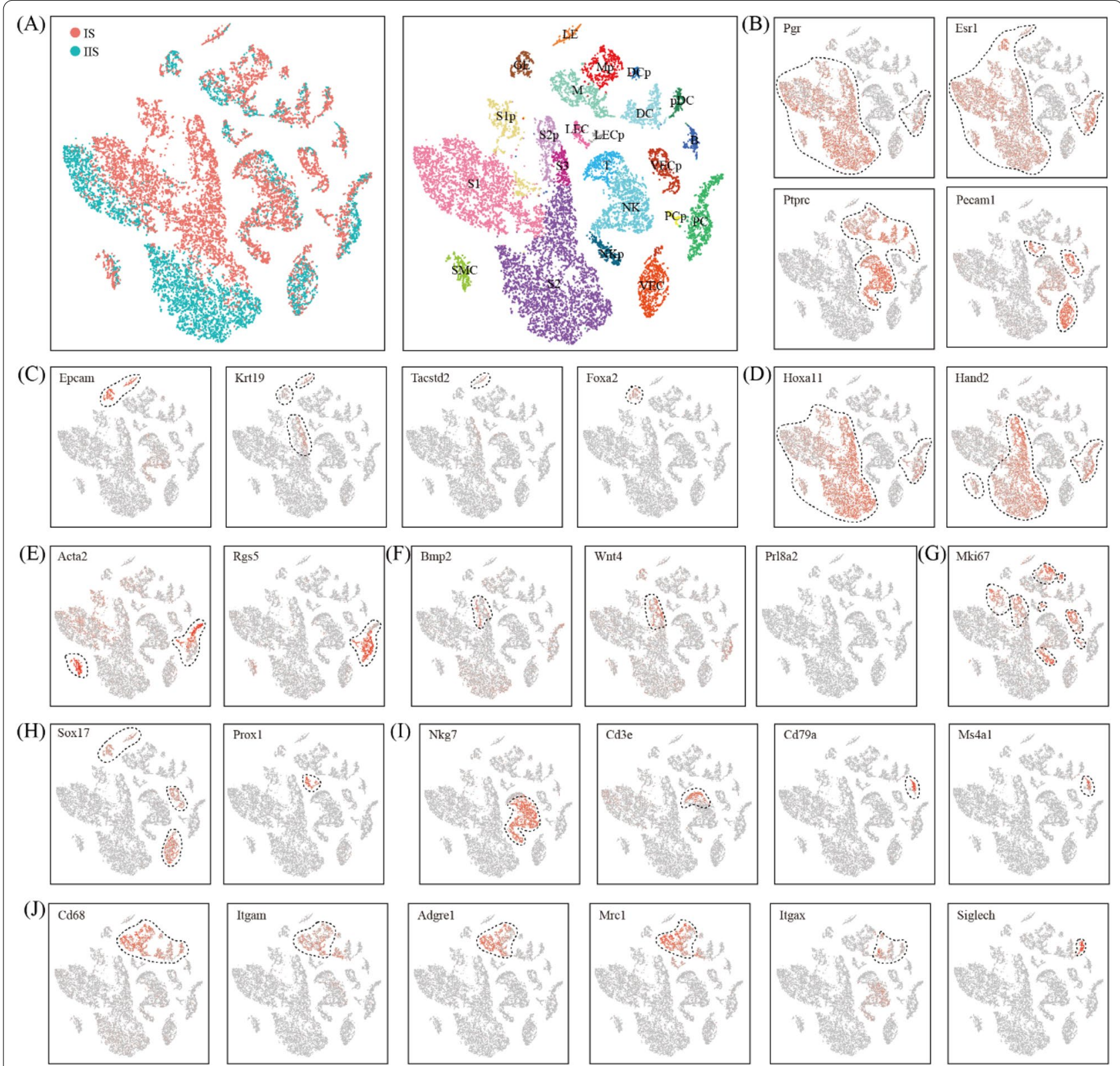

Fig. 2 Identification of different cell types by using canonical marker genes. A The t-Stochastic neighbor embedding (tSNE) representation of single-cell RNA-seq data obtained from IS and IIS of the uterus. Single cells were grouped by cellular origin (right) and cell clusters (left). LE, luminal epithelial cells; GE, glandular epithelial cells; S1, deep stromal cells; S1 p, proliferating deep stromal cells; S2, superficial stromal cells; S2, proliferating superficial stromal cells; S3, primary decidualization zone cells; SMC, smooth muscle cells; PC, pericytes; PCp, proliferating pericytes; VEC, vascular endothelial cells; VECp, proliferating vascular endothelial cells; LEC, lymphatic endothelial cells; LECp, proliferating lymphatic endothelial cells; NK, natural killer cells; NKp, proliferating natural killer cells; T, T cells; B, B cells; M, macrophages; Mp, proliferating macrophages; DC, dendritic cells; DCp, proliferating dendritic cells; pDC, plasmacytoid dendritic cells. (B-J) The expression pattern of canonical marker genes projected onto TSNE plots. Shown are pan-marker genes for hormone-responsive cells, immune cells and endothelial cells $\mathbf{B}$, epithelial cells $\mathbf{C}$, stromal cells $\mathbf{D}$, smooth muscle cells and pericytes $\mathbf{E}$, decidual cells $\mathbf{F}$, proliferating cells $\mathbf{G}$, endothelial cells $\mathbf{H}$, lymphocytes $\mathbf{I}$, and myeloid cells $\mathbf{J}$. Dashed lines denote the boundaries of the cell cluster of interest 
Unsupervised clustering analysis revealed 23 distinct cell clusters for all cells from IIS and IS combined (Fig. 2A). Major cell types were defined using the expression of known cell type-specific genes, with hormone-responsive cells expressing Pgr and Esr1 [24, 25], endothelial cells expressing Pecam1 [26] and immune cells expressing Ptprc [27] (Fig. 2B).

Hormone-responsive cells included epithelial cells expressing Epcam and Krt19 [28] (Fig. 2C), stromal cells expressing Hoxa11 [29] (Fig. 2D), smooth muscle cells expressing Acta2 [11] and pericytes expressing Rgs5 [30] (Fig. 2E). We found 2 epithelial cell clusters, LE and GE. LE was luminal epithelial cells expressing Tacstd2 and GE was glandular epithelial cells expressing Foxa2 [31]. We identified 5 stromal cell clusters, S1, S1p, S2, S2p and S3. Cells in S2 but not S1 expressed high levels of Hand2, implying that S2 was superficial stromal cells and S1 was deep stromal cells [32]. S3 was primary decidual zone stromal cells expressing decidualization marker genes Wnt4 [33] (Fig. 2F). S1p and S2p were a subset of proliferating S1 and S2 with high level of Mki67, respectively (Fig. 2G). Only 1 smooth muscle cell cluster, SMC, was found. Meanwhile, 2 pericyte clusters, PC and its proliferating subset PCp, were identified. Endothelial cells had 4 clusters: VEC and its proliferating subset VECp are vascular endothelial cells expressing Sox17, while LEC and its proliferating subset LECp are lymphatic endothelial cells expressing Prox1 [26] (Fig. 2H). There were 9 immune cell clusters (Fig. 2I, J). Included were natural killer cells (NK, $\mathrm{Ptprc}^{+} \mathrm{Nkg}^{+} \mathrm{Cd} 3 \mathrm{e}^{-}$) [27], proliferating natural killer cells $\left(\mathrm{NKp}, \mathrm{Ptprc}^{+} \mathrm{Nkg}^{-} \mathrm{Cd}^{-} \mathrm{e}^{+} \mathrm{Mki}^{+} 7^{+}\right.$), $\mathrm{T}$ cells (T, Ptprc $\left.{ }^{+} \mathrm{Nkg} 7^{-} \mathrm{Cd} 3 \mathrm{e}^{+}\right)$[27], B cells (B, Ptprc ${ }^{+} \mathrm{Cd} 79 \mathrm{a}^{+} \mathrm{Ms} 4 \mathrm{a} 1^{+}$) [27], macrophages (M, Ptprc ${ }^{+}$Adgre $1^{+}$) [34], proliferating macrophages (M, Ptprc ${ }^{+}$Adgre $1^{+} \mathrm{Mki}^{+} 7^{+}$), dendritic cells (DC, Ptprc $^{+}$Itgax $^{+}$) [34], proliferating dendritic cells (DC, Ptprc $^{+}$Itgax $^{+} \mathrm{Mki}^{+} 7^{+}$) and plasmacytoid dendritic cells $\left(\mathrm{pDC}, \mathrm{Ptprc}^{+}\right.$Siglech $^{+}$) [35].

Finally, we aimed to discover novel markers for each cell type. We selected genes that were expressed significantly higher in the cell type of interest than the other cell types by Wilcoxon rank sum test. A complete list of these marker genes was presented in Additional file 1: Table S1.

\section{Reconstruction of developmental trajectory for primary decidual zone}

In our single-cell RNA-seq data, we identified 5 clusters of stromal cells: S1 (deep stromal cells), S1p (proliferating deep stromal cells), S2 (superficial stromal cells), S2p (proliferating superficial stromal cells) and S3 (primary decidual zone stromal cells, PDZ). We selected signature genes for each cell cluster by using Wilcoxon rank sum test. After the removal of redundancy, we identified a total of 1784 signature genes (Additional file 2: Table S2). Through heatmap, we grouped all these signature genes into 4 gene sets (Fig. 3A). Gene set 1 with 403 genes were S1-specific. Gene ontology analysis showed that these genes were enriched in cell adhesion $\left(\mathrm{P}=4.20 \times 10^{-11}\right)$, developmental processes $\left(\mathrm{P}=1.10 \times 10^{-4}\right)$, cell organization and biogenesis $\left(\mathrm{P}=3.27 \times 10^{-4}\right)$, stress response $\left(\mathrm{P}=4.88 \times 10^{-4}\right)$ and protein metabolism $\left(\mathrm{P}=3.87 \times 10^{-2}\right)$. Gene set 2 with 352 genes were decreased in S3 compared to its intermediate S2p. These genes were enriched in DNA metabolism $\left(\mathrm{P}=1.00 \times 10^{-11}\right)$, cell cycle and proliferation $\left(\mathrm{P}=1.00 \times 10^{-11}\right)$, cell organization and biogenesis $\left(\mathrm{P}=2.59 \times 10^{-11}\right)$ and protein metabolism $\left(\mathrm{P}=3.97 \times 10^{-3}\right)$. Gene set 3 of 300 genes were S2-specific. Based on GO, enriched terms were protein metabolism $\left(\mathrm{P}=3.39 \times 10^{-11}\right)$, developmental processes $\left(\mathrm{P}=6.05 \times 10^{-8}\right)$ and cell cycle \& proliferation $\left(\mathrm{P}=8.09 \times 10^{-3}\right)$. Gene set 4 of 729 genes were unchanged or increased in S3 compared to its intermediate S2p. Enriched GO terms were protein metabolism $\left(\mathrm{P}=2.49 \times 10^{-8}\right)$, RNA metabolism $\left(\mathrm{P}=3.23 \times 10^{-7}\right), \quad$ DNA metabolism $\left(\mathrm{P}=4.42 \times 10^{-5}\right)$, transport $\left(\mathrm{P}=1.96 \times 10^{-3}\right)$ cell organization and biogenesis $\left(\mathrm{P}=2.90 \times 10^{-5}\right)$, and cell cycle and proliferation $\left(\mathrm{P}=4.91 \times 10^{-3}\right)$.

The expression of known marker genes for PDZ (S3) were examined. We found that PDZ expressed panstromal cell markers Pgr, Esr1 and Hoxa11, as well as the superficial stromal cell marker Hand2. Additionally, PDZ expressed decidualization marker Wnt4, but not Prl8a2 [36]. PDZ ceased proliferation from its intermediate S2p, showing low expression of Mki67. Interestingly, although PDZ expressed mesenchymal marker Vim, they were also positive for epithelial marker Krt19 (Fig. 3B).

To further reveal the relationship between these 5 stromal cell clusters, pseudotime trajectory analysis was conducted. Cells were arranged in a pseudotime manner with a pedigree reconstruction algorithm for biological processes based on transcriptional similarity. We found 2 paths of interest: (1) primary decidual zone formation, i.e. S2- > S2p/S3; and (2) secondary decidual zone formation, i.e. S1->S1p (Fig. 3C).

\section{Cell-cell communication between primary decidual zone and trophoblast giant cells}

The cell-cell communication between primary decidual zone and trophoblast giant cells represents the key mechanism of embryo implantation. However, due to the relatively small number of embryonic cells at the implantation site, we did not find any embryo-related cell clusters in our single-cell RNA-seq data. Alternatively, we re-analyzed a published single-cell RNA-seq dataset on 
(A)
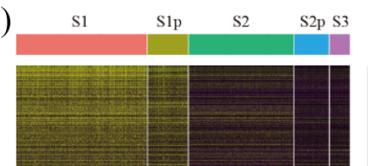

cell adhesion

- developmental processes

- cell organization and biogenesis

- stress response

- protein metabolism

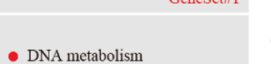

- DNA metabolism

- cell cycle and proliferation

- cell organization and biogenesis

- protein metabolism

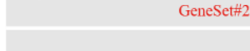

- protein metabolism

- developmental processes

- cell cycle and proliferation

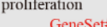

\section{(1)}

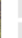

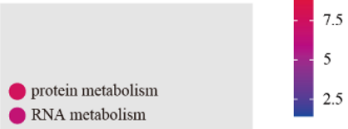

RNA metabolism

- transport

- cell organization and biogenesis

- cell cycle and proliferation

GeneSet/14

$\begin{array}{lllll}-2 & -1 & 0 & 1 & 2\end{array}$

(C)

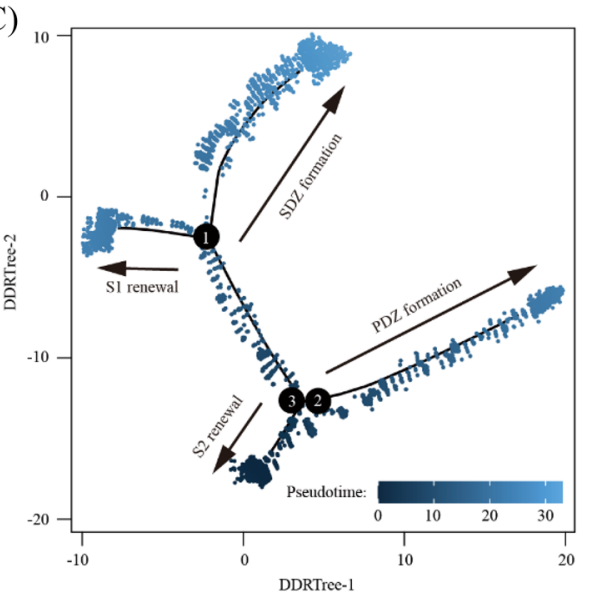

(B)
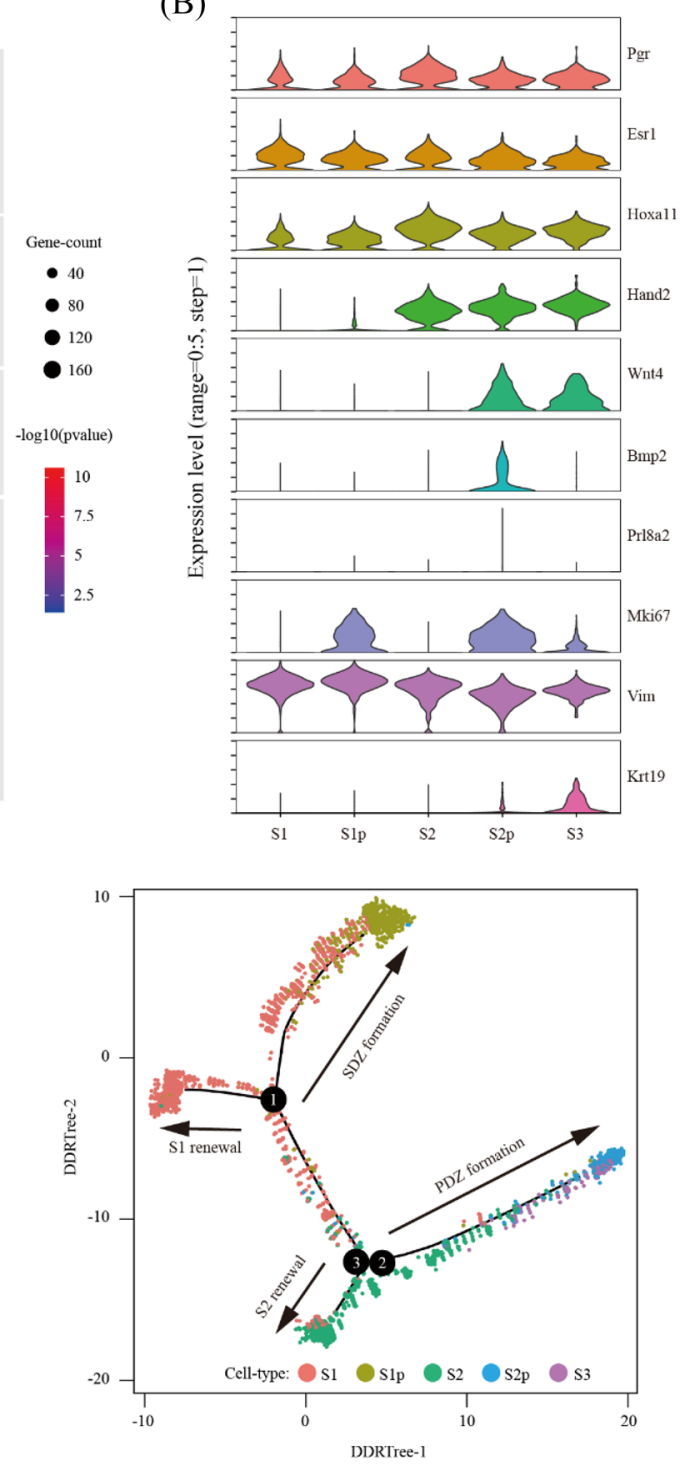

Fig. 3 Developmental trajectory of primary decidual zone. A Heatmap of gene expression signatures for all stromal cell clusters (left). All signature genes were divided into 4 gene sets and enriched gene ontology terms were assigned accordingly (right). B The expression pattern of marker genes for clusters of stromal cell by violin plot. Shown were pan-stromal cell markers (Pgr, Esr1, Hoxa11 and Hand2), decidual cell markers (Wnt4 and Bmp2 and Prl8a2), proliferating cell marker (Mki67), mesenchymal marker (Vim) and epithelial marker (Krt19). C Pseudotime ordering of stromal cells. The distribution of pseudotime (left) and cell type (right) across the reconstructed trajectory were displayed. PDZ, primary decidual zone; SDZ, secondary decidual zone

mouse E5.5 blastocysts [37]. E5.5 is equivalent to gestational day 6 in our study. By using canonical marker genes, 4 major cell types were identified (Fig. 4A). The 4 cell clusters were visceral endoderm (VE) expressing Apob and Amn [38], epiblast (EPI) expressing Pou5f1 and Nanog [39], extraembryonic ectoderm or ectoplacental cone (EXE/EPC) expressing Elf5 and Cdx2 [39], and trophoblast giant cells (TGC) expressing Gata2 [37] and Prl3d1 [40] (Fig. 4B-E).
By using the CellChat software, we predicted the ligand-receptor interactions between PDZ (S3) and TGC. We found a total of 20 ligand-receptor interaction pairs (Fig. 4F). Based on pathway analysis, these ligand-receptor interactions were enriched among ECM-receptor interaction $\left(\mathrm{FDR}=1.00 \times 10^{-18}\right)$, PI3KAkt signaling pathway $\left(\mathrm{FDR}=1.00 \times 10^{-18}\right)$, Regulation of actin cytoskeleton $\left(\mathrm{FDR}=1.26 \times 10^{-9}\right)$, Rap1 signaling pathway $\left(\mathrm{FDR}=1.58 \times 10^{-6}\right)$, Ras signaling 


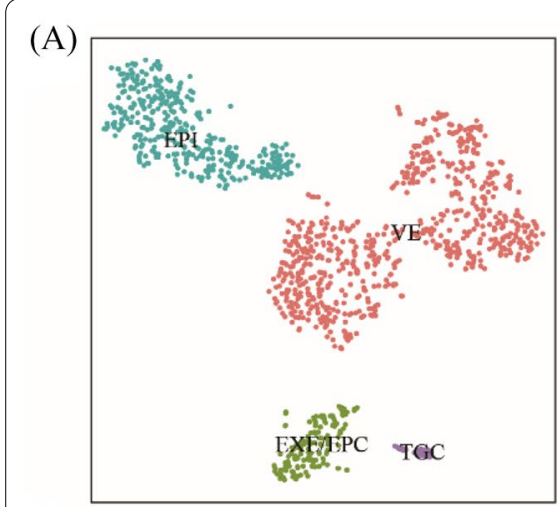

(B)

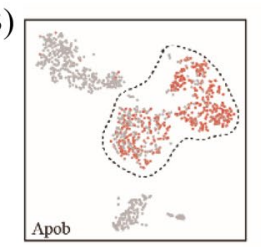

(D)

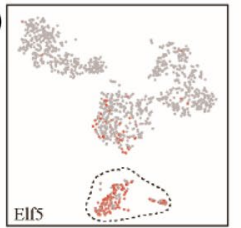

(F)
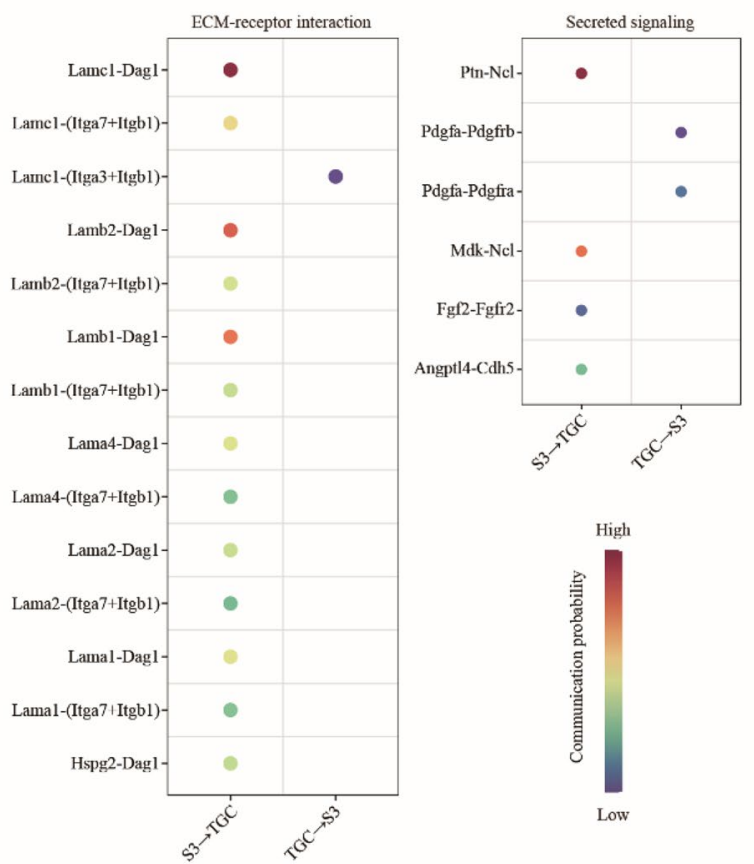
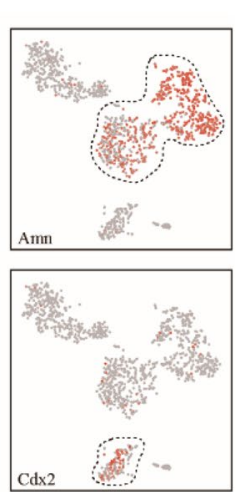

(C)

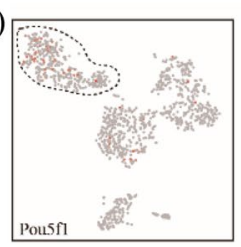

(E)
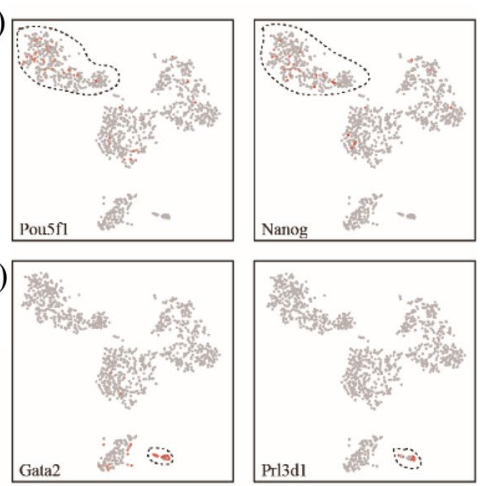

(G)
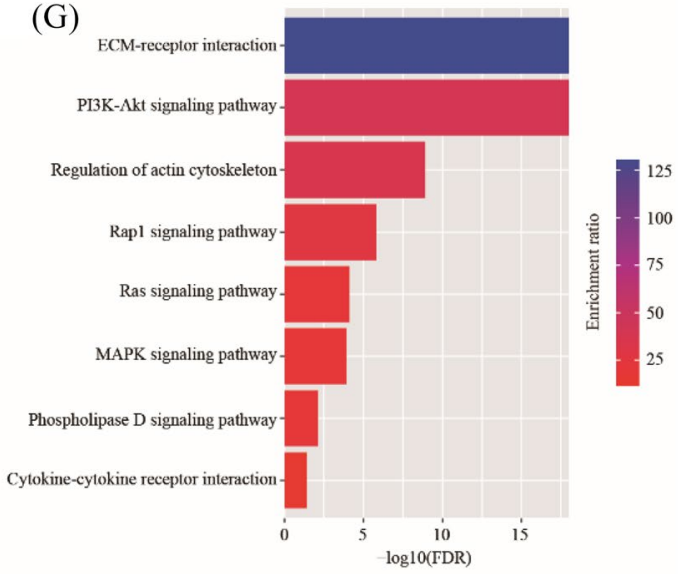

Fig. 4 Cell-cell communication between primary decidual zone and trophoblast giant cells. A A single-cell atlas of E5.5 embryos which were collected from GD6 uterus from a previous study. EPI, epiblast; VE, visceral endoderm; EXE/EPC, extraembryonic ectoderm and ectoplacental cone; TGC, trophoblast giant cells. B-E TSNE map showing the expression pattern of well-known marker genes. F Dot plot showing selected ligand-receptor interactions underlying the crosstalk between primary decidual zone (S3) and trophoblast giant cells (TGC). The communication probability defined by the CellChat software were indicated by color. G KEGG Pathway enrichment analysis of ligand-receptor pairs by using the Metascape online tools

pathway $\left(\mathrm{FDR}=7.94 \times 10^{-5}\right)$, MAPK signaling pathway $\left(\mathrm{FDR}=1.26 \times 10^{-4}\right)$, Phospholipase $\mathrm{D}$ signaling pathway $\left(\mathrm{FDR}=7.94 \times 10^{-3}\right)$ and Cytokine-cytokine receptor interaction $\left(\mathrm{FDR}=3.98 \times 10^{-2}\right)$ (Fig. 4G).

\section{The impact of PDZ stromal cells on other stromal cells}

We investigated the abundance of major stromal cell types at IS compared to IIS. The $\chi^{2}$ test was employed to assess the significance of difference between two groups. By using the criteria of $\mathrm{P}<0.05$ and fold change $>2$, we found that the proportion of S1 was unchanged, whereas the proportion of S2 significantly decreased in IS compared to IIS. Meanwhile, S1p and S2p were almost exclusively detected in IS (Fig. 5A), and so was S3 (0.0\% in IIS vs $3.1 \%$ in IS, data not shown). According to our trajectory analysis, S2p was originated from S2 and S3 was originated from S2p. Surprisingly, the proportion of all these S2-lineage cells $(\mathrm{S} 2+\mathrm{S} 2 \mathrm{p}+\mathrm{S} 3)$ together in IS was still less than that of S2 in IIS (24.6\% vs $36.8 \%)$. This phenomenon was likely due to cell loss at PDZ from GD6. In fact, PDZ disappears by GD8 [41].

We investigated the breadth of transcriptional changes in each stromal cell type by performing differential gene 

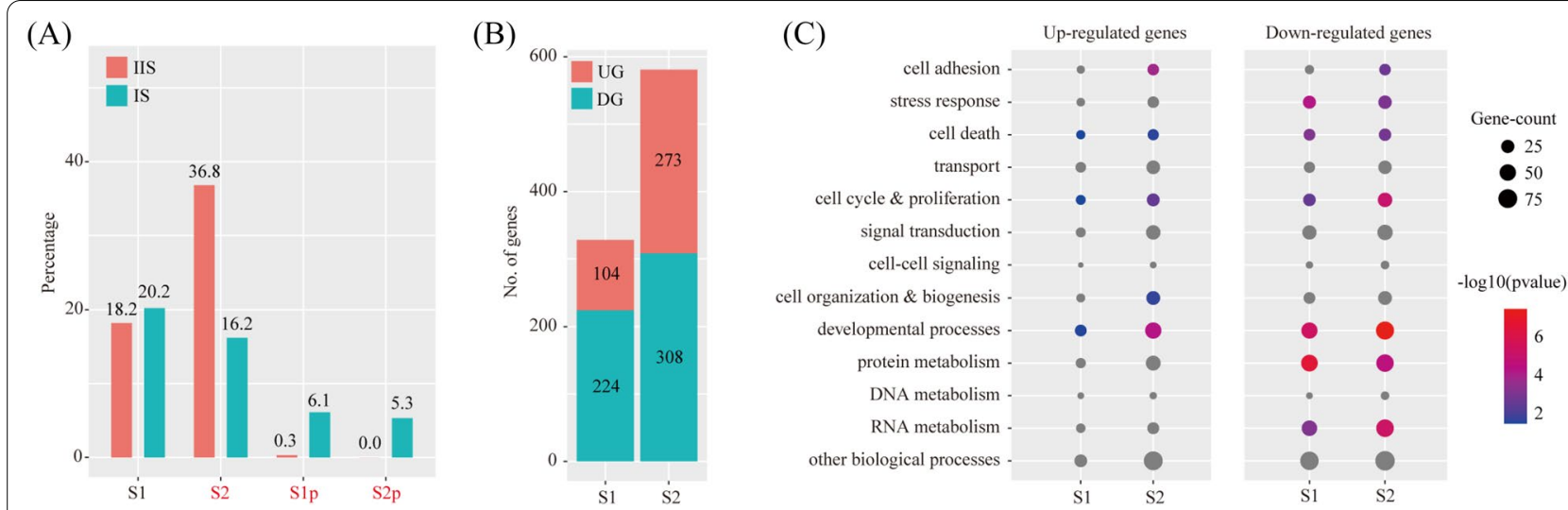

Fig. 5 Cell abundance and gene expression changes in stromal cells upon embryo implantation. A Bar plot showing the cell population change of major stromal cell types (S1 and S2) at IS compared to IIS. Cell types with FC $>2$ and $\mathrm{P}<0.05$ by $\mathrm{X}^{2}$ test were labeled in red. B Bar plot showing the count of differentially expressed genes in each stromal cell type. The threshold values for differentially expressed genes were: logFC $>0.25$ and $P<0.05$. UG, up-regulated gene; DG, down-regulated genes. C Gene ontology (GO) enrichment analysis of differentially expressed genes. Differentially expressed genes were grouped based on MGI GOslim terms under the biological process categories. Up-regulated genes and down-regulated genes were tested separately. Abbreviations for cell types are listed in Fig. 2

expression analysis. Using a $\operatorname{logFC}$ cutoff of 0.25 and a pvalue cutoff of 0.05 , we identified 328 and 581 differentially expressed genes for S1 and S2, respectively (Fig. 5B and Additional file 3: Table S3). We then explored the biological implications of differentially expressed genes using gene ontology (GO) analysis. Our results indicated that similar functional changes occurred during embryo implantation in both S1 and S2 (Fig. 5C).

In order to determine the impact of PDZ (S3) on S1 and S2, we used the CellChat software to predict the ligand-receptor interactions. Only secreted factors from PDZ were considered. We found a total of 35 ligand-receptor interaction pairs (Fig. 6A). Pathway analysis revealed that these ligand-receptor interactions were enriched among Cytokine-cytokine receptor interaction $\left(\mathrm{FDR}=1.26 \times 10^{-10}\right)$, PI3K-Akt signaling pathway $\left(\mathrm{FDR}=2.00 \times 10^{-9}\right)$, Regulation of actin cytoskeleton $\left(\mathrm{FDR}=3.16 \times 10^{-9}\right)$, Hippo signaling pathway $\left(\mathrm{FDR}=3.98 \times 10^{-9}\right)$, ECM-receptor interaction $\left(\mathrm{FDR}=3.98 \times 10^{-8}\right)$, Rap1 signaling pathway $\left(\mathrm{FDR}=1.00 \times 10^{-6}\right), \quad$ MAPK $\quad\left(\mathrm{FDR}=3.16 \times 10^{-6}\right)$, Wnt signaling pathway $\left(\mathrm{FDR}=2.00 \times 10^{-5}\right)$, Ras signaling pathway $\left(\mathrm{FDR}=2.00 \times 10^{-5}\right)$, mTOR signaling pathway $\left(\mathrm{FDR}=2.51 \times 10^{-5}\right)$, TGF-beta signaling pathway $\left(\mathrm{FDR}=2.51 \times 10^{-5}\right)$, Endocytosis $\left(\mathrm{FDR}=6.31 \times 10^{-5}\right)$ and Phagosome $\left(\mathrm{FDR}=7.94 \times 10^{-3}\right)$ (Fig. 6B).

\section{The impact of primary decidual zone stromal cells on immune cells}

By using the criteria of $\mathrm{P}<0.05$ and fold change $>2$, we found that the proportion of NKp, Mp and DCp were significantly increased at IS compared IIS (Fig. 7A). Using a $\operatorname{logFC}$ cutoff of 0.25 and a pvalue cutoff of 0.05 , we identified 286, 294, 302, 495, 263 and 380 differentially expressed genes for $\mathrm{T}, \mathrm{B}, \mathrm{NK}, \mathrm{M}, \mathrm{DC}$ and $\mathrm{pDC}$ respectively (Fig. 7B and Additional file 3: Table S3). Differentially expressed genes were further characterized by GO analysis (Fig. 7C). Our data indicated that each immune cell type invoked distinct biological processes in order to accommodate embryo implantation.

We predicted the ligand-receptor interactions between PDZ (S3) and immune cells (T, B, NK, M, DC and $\mathrm{pDC}$ ) using the CellChat software. We found a total of 26 ligand-receptor interaction pairs (Fig. 8A). Pathway analysis revealed that these ligand-receptor interactions were enriched among Cytokine-cytokine receptor interaction (FDR $=1.58 \times 10^{-7}$ ), PI3K-Akt signaling pathway $\left(\mathrm{FDR}=1.26 \times 10^{-6}\right)$, MAPK signaling pathway $\left(\mathrm{FDR}=3.16 \times 10^{-4}\right)$, Endocytosis $\left(\mathrm{FDR}=3.98 \times 10^{-4}\right)$, ECM-receptor interaction $\left(\mathrm{FDR}=3.98 \times 10^{-4}\right)$, TGFbeta signaling pathway $\left(\mathrm{FDR}=3.98 \times 10^{-4}\right), \quad$ Rap1 signaling pathway $\left(\mathrm{FDR}=7.94 \times 10^{-4}\right)$, Hippo signaling pathway $\left(\mathrm{FDR}=2.51 \times 10^{-3}\right)$, Regulation of actin cytoskeleton $\left(\mathrm{FDR}=7.94 \times 10^{-3}\right)$, Toll-like receptor signaling pathway $\left(\mathrm{FDR}=1.00 \times 10^{-2}\right)$, Ras signaling pathway $\left(F D R=1.26 \times 10^{-2}\right)$, Natural killer cell mediated cytotoxicity $\left(\mathrm{FDR}=1.58 \times 10^{-2}\right)$, Jak-STAT signaling pathway $\left(\mathrm{FDR}=3.16 \times 10^{-2}\right)$ and NOD-like receptor signaling pathway $\left(\mathrm{FDR}=3.98 \times 10^{-2}\right.$ ) (Fig. $\left.8 \mathrm{~B}\right)$.

\section{The impact of primary decidual zone on endothelial cells}

By using the criteria of $\mathrm{P}<0.05$ and fold change $>2$, we found that the proportions of both VEC and LEC were significantly decreased in IS compared to IIS, which is in 


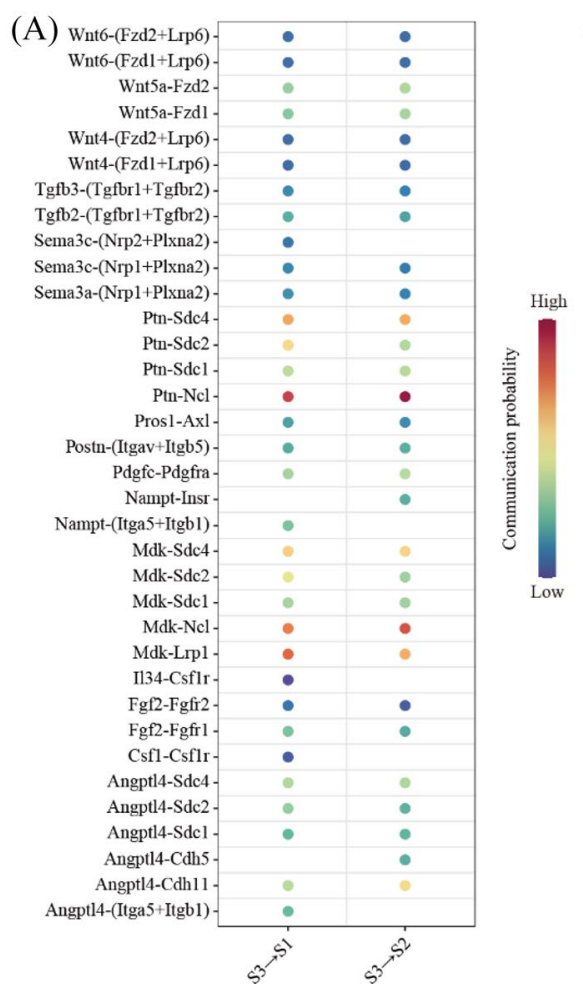

(B)

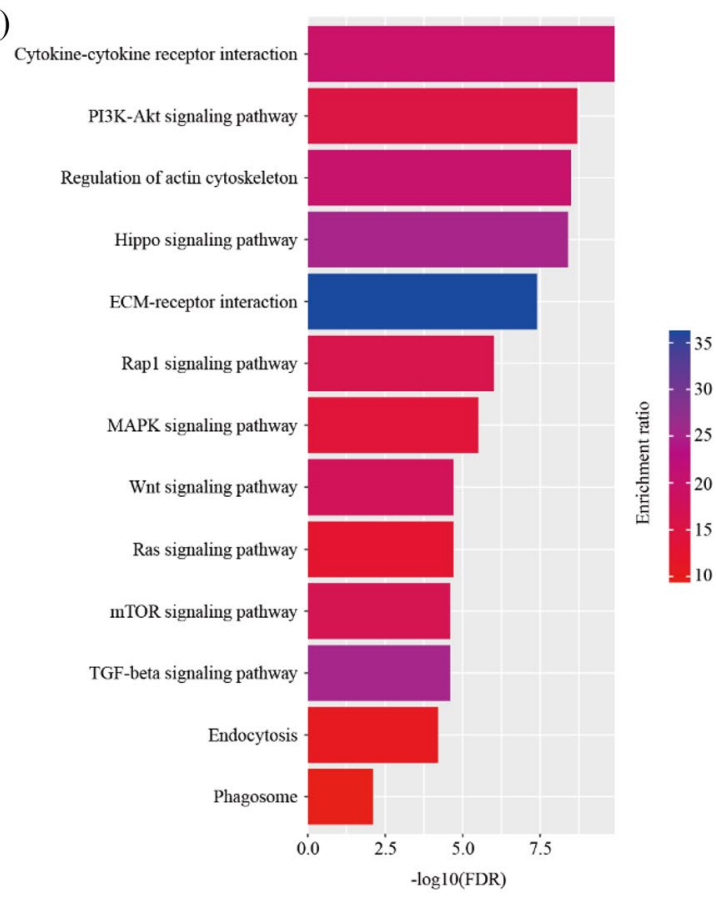

Fig. 6 The impact of primary decidual zone stromal cells on other stromal cells. A Dot plot showing selected ligand-receptor interactions underlying the crosstalk between primary decidual zone stromal cells (S3) and other stromal cells (S1 and S2). The communication probability defined by the CellChat software were indicated by color. B KEGG Pathway enrichment analysis of ligand-receptor pairs by using the Metascape online tools

line with the fact that PDZ is avascular [42-44]. Interestingly, the proportion of VECp was significantly increased in IS compared to IIS, indicating that the PDZ is inducer of angiogenesis in the uterus (Fig. 9A). Using a $\operatorname{logFC}$ cutoff of 0.25 and a P value cutoff of 0.05 , we identified 263 and 404 differentially expressed genes for VEC and LEC, respectively (Fig. 9B and Additional file 3: Table S3). These differentially expressed genes were further characterized by GO analysis (Fig. 9C).

We predicted the ligand-receptor interactions between PDZ (S3) and VEC/LEC. We found a total of 34 ligand-receptor interaction pairs (Fig. 10A). Pathway analysis revealed that these ligand-receptor interactions were enriched among PI3K-Akt signaling pathway $\left(\mathrm{FDR}=1.00 \times 10^{-23}\right)$, Rap 1 signaling pathway $\left(\mathrm{FDR}=1.00 \times 10^{-16}\right)$, Ras signaling pathway $\left(\mathrm{FDR}=1.00 \times 10^{-14}\right)$, Cytokine-cytokine receptor interaction $\left(\mathrm{FDR}=1.00 \times 10^{-14}\right)$, HIF-1 signaling pathway $\left(\mathrm{FDR}=1.26 \times 10^{-7}\right)$, Hippo signaling pathway $\left(\mathrm{FDR}=1.00 \times 10^{-6}\right)$, Endocytosis $\left(\mathrm{FDR}=3.16 \times 10^{-6}\right)$, Regulation of actin cytoskeleton $\left(\mathrm{FDR}=6.31 \times 10^{-6}\right)$, mTOR signaling pathway $\left(\mathrm{FDR}=1.58 \times 10^{-5}\right)$, MAPK signaling pathway $\left(\mathrm{FDR}=1.58 \times 10^{-5}\right), \quad \mathrm{ECM}$-receptor interaction $\quad\left(\mathrm{FDR}=3.98 \times 10^{-4}\right), \quad$ TGF-beta signaling pathway $\left(\mathrm{FDR}=3.98 \times 10^{-4}\right)$, NOD-like receptor signaling pathway $\left(\mathrm{FDR}=5.01 \times 10^{-3}\right)$, Toll-like receptor signaling pathway $\left(\mathrm{FDR}=1.26 \times 10^{-2}\right)$, AMPK signaling pathway $\left(\mathrm{FDR}=2.00 \times 10^{-2}\right)$, Wnt signaling pathway $\left(\mathrm{FDR}=3.16 \times 10^{-2}\right)$ and Jak-STAT signaling pathway $\left(\mathrm{FDR}=3.98 \times 10^{-2}\right)($ Fig. 10B $)$.

\section{Discussion}

Embryo implantation is a crucial step for human embryo implantation. Previously, we performed single-cell RNA analysis of the mouse uterus during the apposition phase and attachment phase of embryo implantation on gestational days (GD) $4-5[16,17]$. Here, by using the mouse as an animal model, we profiled the single-cell transcriptome for 16,257 cells from mouse uterus at the invasion phase on GD6. We revealed 23 distinct cell clusters, including 5 stromal cell clusters, 2 epithelial cell clusters, 1 smooth muscle cell cluster, 2 pericyte clusters, 4 endothelial cell clusters, and 9 immune cell clusters. To the best of our knowledge, the present study is the first to highlight the transcriptome landscape associated with 

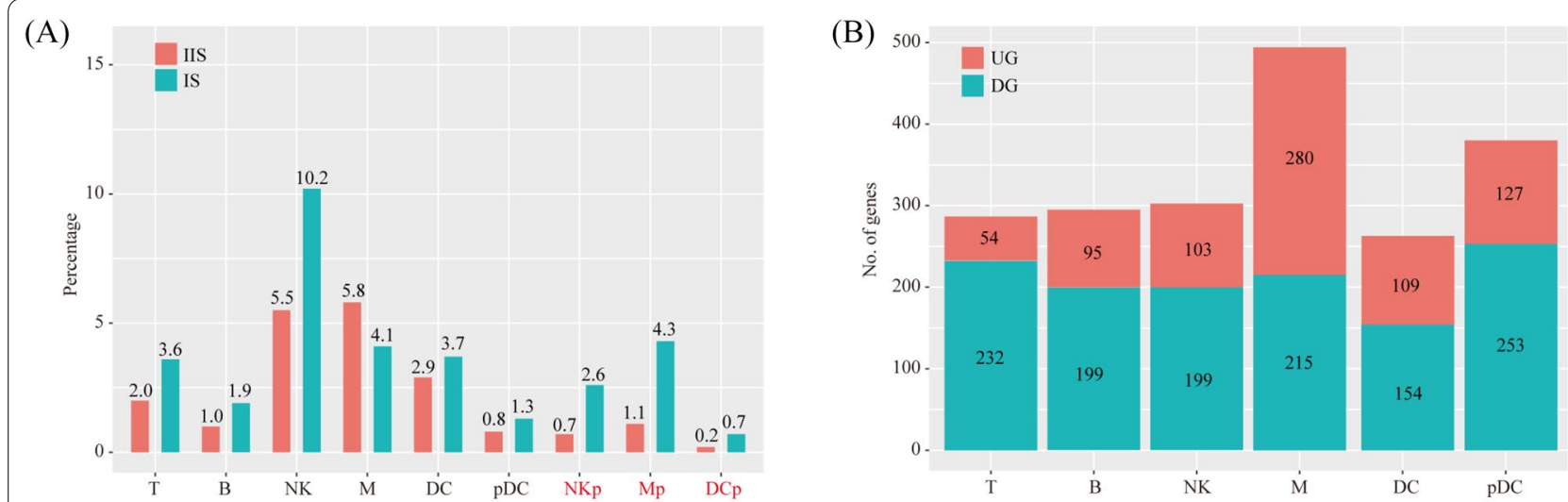

(C)
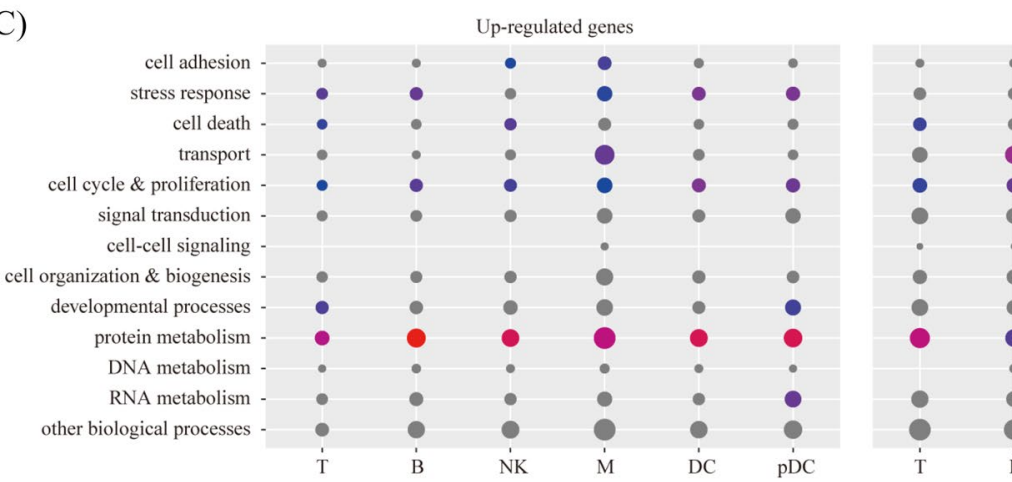

$\begin{array}{ll} & \text { Dow } \\ : & \\ 0 \\ 0 \\ 0 \\ 0 \\ 0 \\ \text { B }\end{array}$
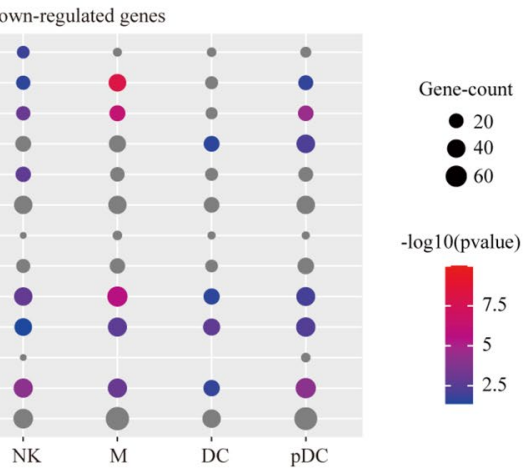

Fig. 7 Cell abundance and gene expression changes in immune cells upon embryo implantation. A Bar plot showing the cell population change of major immune cell types (T, NK, B, M, DC and pDC) at IS compared to IIS. Cell types with FC $>2$ and $\mathrm{P}<0.05$ by $\mathrm{X}^{2}$ test were labeled in red. B Bar plot showing the count of differentially expressed genes in each immune cell type. The threshold values for differentially expressed genes were: $\log F C>0.25$ and $P<0.05$. UG, up-regulated gene; DG, down-regulated genes. C Gene ontology (GO) enrichment analysis of differentially expressed genes. Differentially expressed genes were grouped based on MGI GOslim terms under the biological process categories. Up-regulated genes and down-regulated genes were tested separately. Abbreviations for cell types are listed in Fig. 2

the invasion phase of embryo implantation at single-cell resolution.

In order to accommodate embryo implantation, an important change in mouse uterus is the formation of primary decidual zone (PDZ). The first sign of PDZ formation occurs on the afternoon of GD5. PDZ is fully established by GD6 [45-47]. In this study, we found that the stromal cells at IIS can be divided into 2 cell types, superficial stromal cells expressing Hand2 and deep stromal cells which are negative for Hand2. Through pseudotime trajectory analysis, we confirmed that PDZ at IS was derived from superficial stromal cells. Superficial stromal cells start proliferate to become intermediate PDZ cells with high expression of Wnt4, Bmp2 and Mki67. Notably, Prl8a2, the marker for secondary decidual zone [36], was not expressed. These cells ceased proliferation and became PDZ cells [41]. The expression of another decidual marker Bmp2 [48] was reduced during this process. Interestingly, although PDZ cells expressed mesenchymal marker Vim, they were also positive for epithelial marker Krt19. Our result is in line with previous findings showing that PDZ is avascular and epithelioid in nature [42-44]. In fact, PDZ is thought to function as a partial permeability barrier to safeguard the implanting embryo, as only molecules smaller than $45 \mathrm{kDa}$ are freely permeable though PDZ [49].

The trophectoderm (TE) of mouse embryo is created by the end of the pre-implantation period. After implantation, TE develops into two different types, polar TE and mural TE. Polar TE differentiates into extraembryonic ectoderm (ExE) and ectoplacental cone (EPC), while mural TE differentiates into trophoblast giant cells (TGC). TGC cells are in closest contact to the uterus during embryo implantation [50]. Thus, the interaction between TGC and PDZ represents the key mechanism of embryo implantation. Notably, PDZ cells expressed Ptn and Mdk, while the corresponding receptor $\mathrm{Ncl}$ was expressed in TGC. Currently, little is known about the role of Ptn and Mdk in regulating embryo implantation. Additionally, we found that Fgf2 and Angptl4 expressed in PDZ might function via their receptors Fgfr2 and Cdh5 expressed in TGC. In mouse uterus, Fgf2 in the 


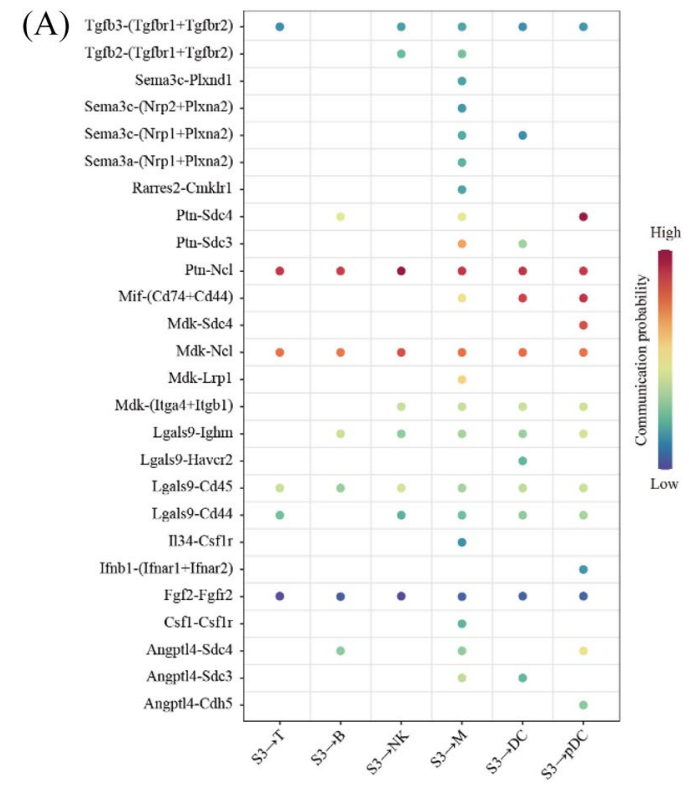

(B)

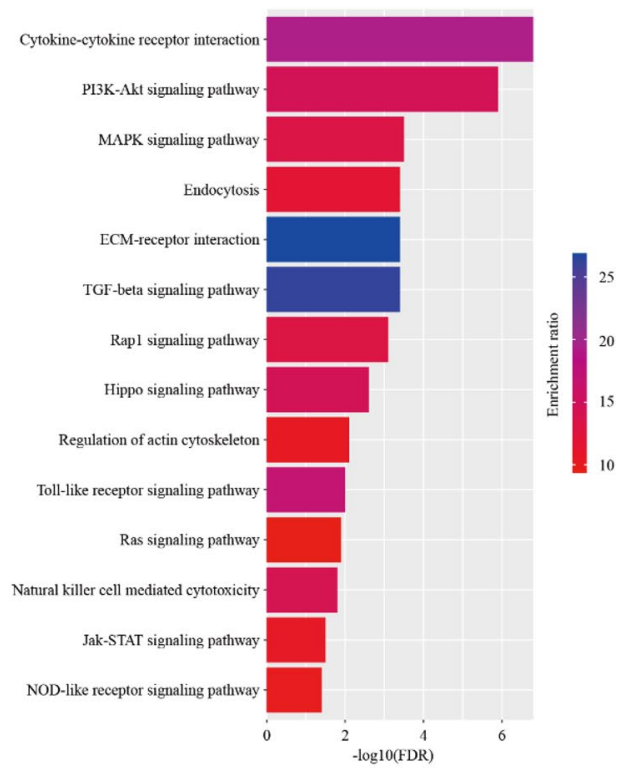

Fig. 8 The impact of primary decidualization zone cells on immune cells. A Dot plot showing selected ligand-receptor interactions underlying crosstalk between primary decidualization zone cells (S3) and immune cells (T, NK, B, M, DC and pDC). The communication probability defined by the CellChat software were indicated by color. B KEGG Pathway enrichment analysis of ligand-receptor pairs by using the Metascape online tools
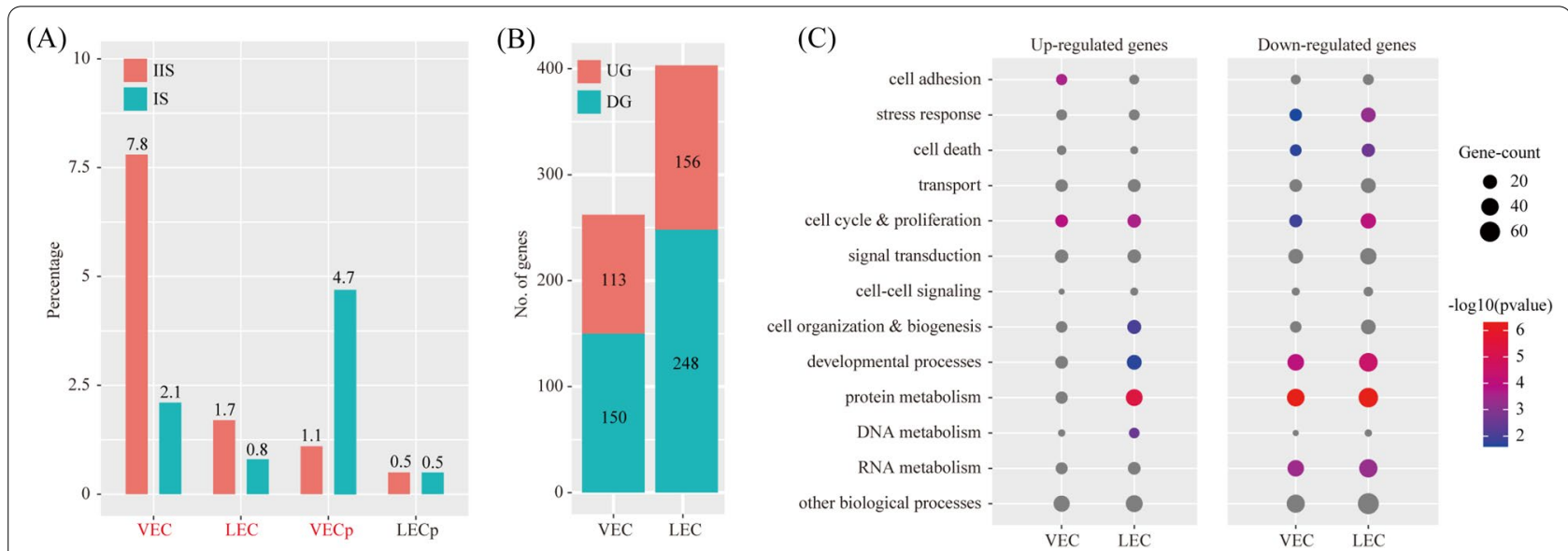

Fig. 9 Cell abundance and gene expression changes in endothelial cells upon embryo implantation. A Bar plot showing the cell population change of major endothelial cell types (VEC and LEC) at IS compared to IIS. Cell types with FC $>2$ and $\mathrm{P}<0.05$ by $\mathrm{X}^{2}$ test were labeled in red. B Bar plot showing the count of differentially expressed genes in each immune cell type. The threshold values for differentially expressed genes were: $\log F C>0.25$ and $P<0.05$. UG, up-regulated gene; DG, down-regulated genes. C Gene ontology (GO) enrichment analysis of differentially expressed genes. Differentially expressed genes were grouped based on MGI GOslim terms under the biological process categories. Up-regulated genes and down-regulated genes were tested separately

superficial stromal cells maintains proliferation of epithelial cells [32]. Because Fgfr2 is also expressed in TGC, Fgf2 in PDZ might contribute to the proliferation of TGC. Angptl4 acts as a secretory protein to regulate angiogenesis in various tissues including the uterus [51]. Vascular endothelial cadherin Cdh5 is also expressed trophoblast cells [52]. Our findings supported the findings showing that predecidual stromal cells have distinctive characteristics of pericytes [53] and trophoblasts mimic the endothelial cells [54]. On the other hand, TGC cells expressed Pdgfa, while the corresponding receptors Pdgfra and Pdgfrb were expressed in PDZ. Indeed, proteome profiling confirmed the presence of Pdgfa in trophoblast supernatant [55]. Pdgfra and Pdgfrb are regarded 
(A)

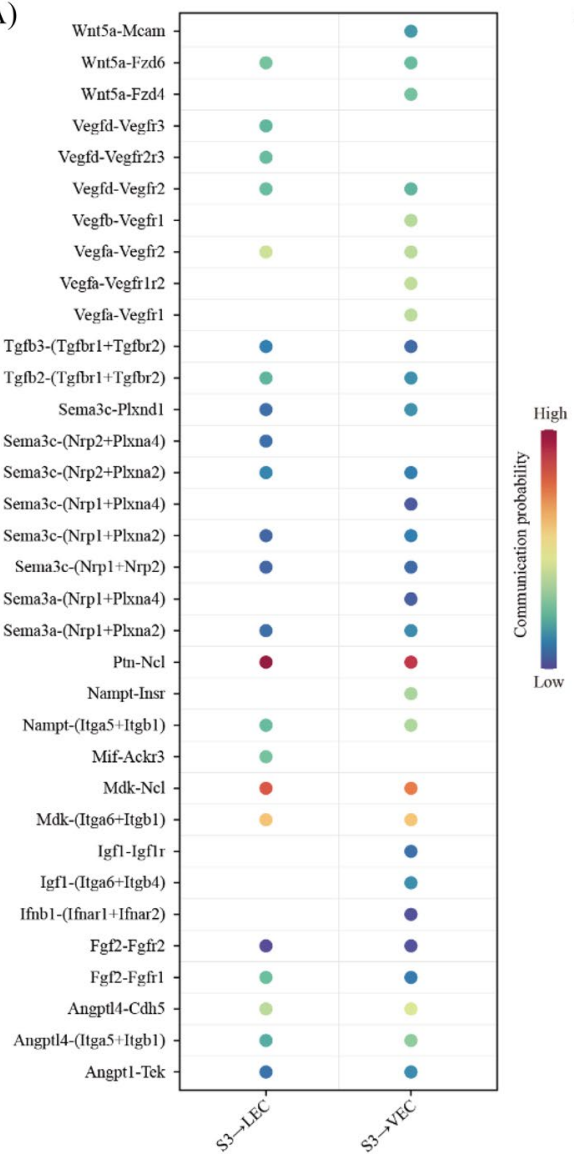

(B)

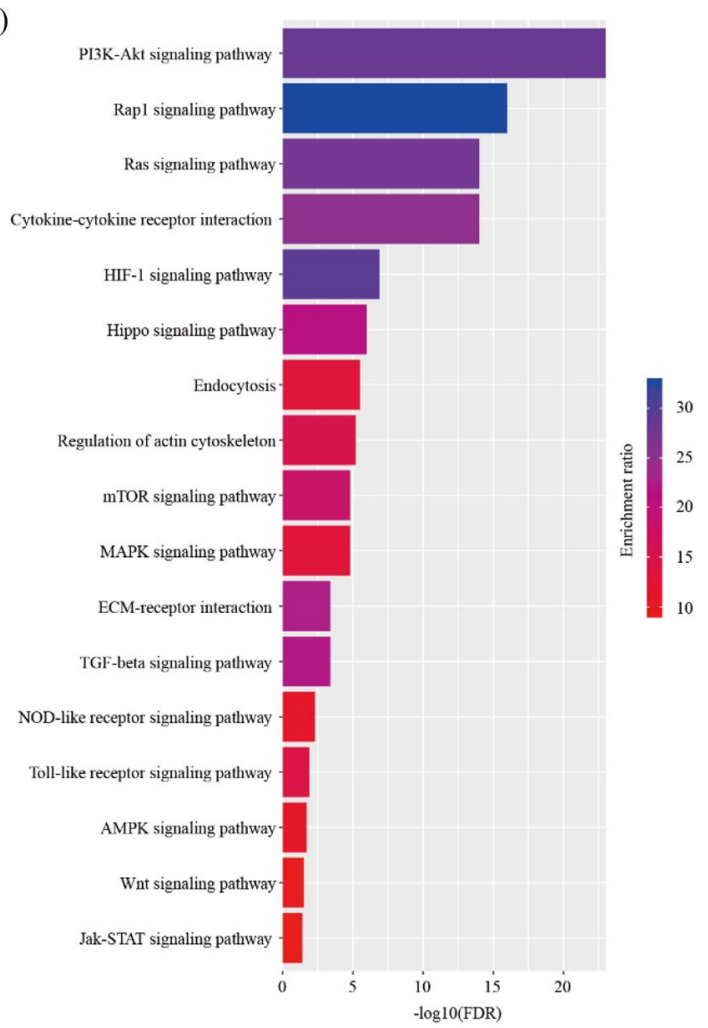

Fig. 10 The impact of primary decidual zone on endothelial cells. A Dot plot showing selected ligand-receptor interactions underlying the crosstalk between primary decidualization zone cells (S3) and endothelial cells (VEC and LEC). The communication probability defined by the CellChat software were indicated by color. B KEGG Pathway enrichment analysis of ligand-receptor pairs by using the Metascape online tools

as canonical cell markers for uterine stromal cells [30]. Therefore, TGC might regulate proliferation and differentiation of PDZ via Pdgfa. Apart from secreted signaling, we found that TGC might also crosstalk with PDZ through ECM-receptor interaction. Our data provided clues for the mechanisms underlying embryo implantation from the aspect of cell-cell communication.

Besides the emergence of PDZ, by comparing IS with IIS, we found apparent cell type abundance changes upon embryo implantation. In particular, the proliferating subsets of S1, S2, NK, M, DC and VEC were significantly increased. There were also massive gene expression changes in these cell types. Considering spatial relationships between cell types, uterine cell types other than PDZ are unable to directly communicate with embryonic cells during embryo implantation. Therefore, these changes were likely caused by PDZ indirectly via secreted signaling. Indeed, we found that many soluble factors from PDZ, such as Wnt4, Wnt5a and Wnt6, might have an influence on S1 and S2 cells. Wnt4 is most abundant in the decidual cells.
In uterus-specific Wnt4 knockout mice, the embryos were able to attach to the uterine luminal epithelium, but they failed to successfully invade into the uterine stroma [33]. During embryo implantation, Wnt5a is highly localized in stromal cells at the mesometrial pole. Mice with uterine inactivation of Wnt5a show impaired embryo implantation and decidualization [47]. Using Wnt6-null mice, it has been shown that Wnt6 is critical for normal stromal cell proliferation during decidualization [56]. The idea that Wnt signaling might act in a paracrine way between PDZ and other stromal cells deserves further investigation. Additionally, PDZ-secreted Tgfb2 and Tgfb3 might target immune cells. Immune cells accumulate at embryo implantation sites and some of them such as DC cells play an important role in embryo implantation [57]. Increasing evidence supports the involvement of TGF $\beta$ signaling in uterine function including embryo implantation [58]. However, whether the TGF $\beta$-mediated PDZ-DC interaction is functional during embryo implantation is still unknown. Moreover, we found that PDZ-secreted Vegfa, Vegfb and 
Vegfd might target endothelial cells. PDZ is avascular, but angiogenesis is accompanied with the emergence of SDZ. It has been well established that uterine immune cells are responsible to angiogenesis in SDZ [59]. Thus, we suggested that PDZ, by synthesizing VEGFs, might be another inducer of angiogenesis besides immune cells. Altogether, our data highlighted the role of PDZ as a key mediator for uterine response in different cell types during embryo implantation.

In conclusion, this study provided a comprehensive single-cell transcriptome atlas for mouse uterus at the invasion phase of embryo implantation. Our data present a valuable resource for deciphering the molecular mechanism underlying embryo implantation.

\section{Supplementary Information}

The online version contains supplementary material available at https://doi. org/10.1186/s13578-022-00749-y.

Additional file 1: Table S1. The complete list of novel and known marker genes for each cell type.

Additional file 2: Table S2. The complete list of signature genes for stromal cell clusters.

Additional file 3: Table S3. The complete list of differentially expressed genes in IS compared to IIS for all cell types $(\log F C>0.25$ and $P<0.05)$

\section{Acknowledgements}

Not applicable.

\section{Authors' contributions}

J-LL supervised the study. J-LL designed the experiments. J-PH performed the experiments. J-PH, QT, Q-YZ and J-LL analyzed the data. J-PH and J-LL wrote the paper. All authors read and approved the final manuscript.

\section{Funding}

This research was funded by National Natural Science Foundation of China (32070845 and 31771665), Guangdong Natural Science Funds for Distinguished Young Scholars (2021B1515020079), Innovation Team Project of Guangdong University (2019KCXTD001), and Guangdong Special Support Program (2019BT02Y276).

\section{Availability of data and materials}

The datasets used and/or analysed during the current study are available from the corresponding author on reasonable request.

\section{Declarations}

Ethics approval and consent to participate Not applicable.

\section{Consent for publication}

Not applicable.

\section{Competing interests}

The authors declare that they have no competing interests.

Received: 31 May 2021 Accepted: 18 January 2022

Published online: 05 February 2022

\section{References}

1. Lee KY, DeMayo FJ. Animal models of implantation. Reproduction. 2004:128:679-95.

2. Tantbirojn P, Crum CP, Parast MM. Pathophysiology of placenta creta: the role of decidua and extravillous trophoblast. Placenta. 2008;29:639-45.

3. Huppertz B. Placental origins of preeclampsia: challenging the current hypothesis. Hypertension. 2008:51:970-5.

4. Ferretti C, Bruni L, Dangles-Marie V, Pecking AP, Bellet D. Molecular circuits shared by placental and cancer cells, and their implications in the proliferative, invasive and migratory capacities of trophoblasts. Hum Reprod Update. 2007;13:121-41.

5. Kshitiz A, Afzal J, Maziarz JD, Hamidzadeh A, Liang C, Erkenbrack EM, et al. Evolution of placental invasion and cancer metastasis are causally linked. Nat Ecol Evol. 2019:3:1743-53.

6. Wang H, Dey SK. Roadmap to embryo implantation: clues from mouse models. Nat Rev Genet. 2006;7:185-99.

7. Cha J, Sun X, Dey SK. Mechanisms of implantation: strategies for successful pregnancy. Nat Med. 2012;18:1754-67.

8. Zhang $\mathrm{S}$, Lin H, Kong S, Wang S, Wang H, Wang H, et al. Physiological and molecular determinants of embryo implantation. Mol Aspects Med. 2013;34:939-80.

9. Nehar-Belaid D, Courau T, Derian N, Florez L, Ruocco MG, Klatzmann D. Regulatory $T$ cells orchestrate similar immune evasion of fetuses and tumors in mice. J Immunol. 2016;196:678-90.

10. Moreno-Moya JM, Franchi NA, Martinez-Escribano S, Martinez-Conejero JA, Bocca S, Oehninger S, et al. Transcriptome of early embryonic invasion at implantation sites in a murine model. Reprod Fert Develop. 2015:9:78.

11. Mucenski ML, Mahoney R, Adam M, Potter AS, Potter SS. Single cell RNAseq study of wild type and Hox 9,10,11 mutant developing uterus. Sci Rep. 2019;9:4557

12. Svensson V, Vento-Tormo $R$, Teichmann SA. Exponential scaling of singlecell RNA-seq in the past decade. Nat Protoc. 2018;13:599-604.

13. Trapnell C, Pachter L, Salzberg SL. TopHat: discovering splice junctions with RNA-Seq. Bioinformatics. 2009;25:1105-11.

14. Trapnell C, Williams BA, Pertea G, Mortazavi A, Kwan G, van Baren MJ, et al Transcript assembly and quantification by RNA-Seq reveals unannotated transcripts and isoform switching during cell differentiation. Nat Biotechnol. 2010:28:511-5

15. Zhao M, Zhang WQ, Liu JL. A study on regional differences in decidualization of the mouse uterus. Reproduction. 2017;153:645-53.

16. Yang Y, He JP, Liu JL. Cell-Cell Communication at the Embryo Implantation Site of Mouse Uterus Revealed by Single-Cell Analysis. Int J Mol Sci. 2021;22:8.

17. Yang Y, Zhu QY, Liu JL. Deciphering mouse uterine receptivity for embryo implantation at single-cell resolution. Cell Prolif. 2021;9:e13128.

18. Stuart T, Butler A, Hoffman P, Hafemeister C, Papalexi E, Mauck WM 3rd, et al. Comprehensive integration of single-cell data. Cell. 2019;177:1888-902

19. Qiu X, Hill A, Packer J, Lin D, Ma YA, Trapnell C. Single-cell mRNA quantification and differential analysis with Census. Nat Methods. 2017; 14:309-15.

20. Liu JL, Zuo RJ, Peng Y, Fu YS. The impact of multiparity on uterine gene expression and decidualization in mice. Reprod Sci. 2016:23:687-94.

21. Law M, Shaw DR. Mouse genome informatics (MGl) is the international resource for information on the laboratory mouse. Methods Mol Biol. 2018;1757:141-61.

22. Zhou Y, Zhou B, Pache L, Chang M, Khodabakhshi AH, Tanaseichuk O, et al. Metascape provides a biologist-oriented resource for the analysis of systems-level datasets. Nat Commun. 2019;10:1523.

23. Jin S, Guerrero-Juarez CF, Zhang L, Chang I, Ramos R, Kuan CH, et al. Inference and analysis of cell-cell communication using Cell Chat. Nat Commun. 2021:12:1088

24. Hamilton KJ, Arao Y, Korach KS. Estrogen hormone physiology: reproductive findings from estrogen receptor mutant mice. Reprod Biol. 2014;14:3-8.

25. Wu SP, Li R, DeMayo FJ. Progesterone receptor regulation of uterine adaptation for pregnancy. Trends Endocrinol Metab. 2018;29:481-91. 
26. Kalucka J, de Rooij L, Goveia J, Rohlenova K, Dumas SJ, Meta E, et al. Single-cell transcriptome atlas of murine endothelial cells. Cell. 2020:180:764-79.

27. Hilton $H G$, Rubinstein ND, Janki P, Ireland AT, Bernstein N, Fong NL, et al. Single-cell transcriptomics of the naked mole-rat reveals unexpected features of mammalian immunity. PLoS Biol. 2019;17:e3000528.

28. Jin S. Bipotent stem cells support the cyclical regeneration of endometrial epithelium of the murine uterus. Proc Natl Acad Sci USA. 2019:116:6848-57.

29. Gendron RL, Paradis H, Hsieh-Li HM, Lee DW, Potter SS, MarkoffE. Abnormal uterine stromal and glandular function associated with maternal reproductive defects in Hoxa-11 null mice. Biol Reprod. 1997:56:1097-105.

30. Kirkwood PM, Gibson DA, Smith JR, Wilson-Kanamori JR, Kelepouri O, Esnal-Zufiaurre A, et al. Single-cell RNA sequencing redefines the mesenchymal cell landscape of mouse endometrium. FASEB J. 2021;35:e21285.

31. Fu DJ, De Micheli AJ, Bidarimath M, Ellenson LH, Cosgrove BD, FleskenNikitin A, et al. Cells expressing PAX8 are the main source of homeostatic regeneration of adult mouse endometrial epithelium and give rise to serous endometrial carcinoma. Dis Models Mech. 2020:13:8.

32. Li Q, Kannan A, DeMayo FJ, Lydon JP, Cooke PS, Yamagishi H, et al. The antiproliferative action of progesterone in uterine epithelium is mediated by Hand2. Science. 2011;331:912-6.

33. Franco HL, Dai D, Lee KY, Rubel CA, Roop D, Boerboom D, et al. WNT4 is a key regulator of normal postnatal uterine development and progesterone signaling during embryo implantation and decidualization in the mouse. FASEB J. 2011;25:1176-87.

34. Keenihan SN, Robertson SA. Diversity in phenotype and steroid hormone dependence in dendritic cells and macrophages in the mouse uterus. Biol Reprod. 2004;70:1562-72.

35. Ye Y, Gaugler B, Mohty M, Malard F. Plasmacytoid dendritic cell biology and its role in immune-mediated diseases. Clin Transl Immunol. 2020;9:e1139.

36. Alam SM, Konno T, Soares MJ. Identification of target genes for a prolactin family paralog in mouse decidua. Reproduction. 2015;149:625-32.

37. Amadei G, Lau KYC, De Jonghe J, Gantner CW, Sozen B, Chan C, et al. Inducible stem-cell-derived embryos capture mouse morphogenetic events in vitro. Develop Cell. 2021;56:366-82

38. Cheng S, Pei Y, He L, Peng G, Reinius B, Tam PPL, et al. Single-Cell RNASeq Reveals Cellular Heterogeneity of Pluripotency Transition and $X$ Chromosome Dynamics during Early Mouse Development. Cell Rep. 2019;26:2593-607.

39. Pfister S, Steiner KA, Tam PP. Gene expression pattern and progression of embryogenesis in the immediate post-implantation period of mouse development. Gene Expr Patterns. 2007; 7: 558-73.

40. Hu D, Cross JC. Development and function of trophoblast giant cells in the rodent placenta. Int J Dev Biol. 2010;54:341-54

41. Tan J, Raja S, Davis MK, Tawfik O, Dey SK, Das SK. Evidence for coordinated interaction of cyclin D3 with p21 and cdk6 in directing the development of uterine stromal cell decidualization and polyploidy during implantation. Mech Dev. 2002;111:99-113.

42. Paria BC, Zhao X, Das SK, Dey SK, Yoshinaga K. Zonula occludens-1 and E-cadherin are coordinately expressed in the mouse uterus with the initiation of implantation and decidualization. Dev Biol. 1999;208:488-501.

43. Parr MB, Parr EL. Permeability of the primary decidual zone in the rat uterus: studies using fluorescein-labeled proteins and dextrans. Biol Reprod. 1986;34:393-403.

44. Tung HN, Parr MB, Parr EL. The permeability of the primary decidual zone in the rat uterus: an ultrastructural tracer and freeze-fracture study. Biol Reprod. 1986;35:1045-58.

45. Li Y, Dewar A, Kim YS, Dey SK, Sun X. Pregnancy success in mice requires appropriate cannabinoid receptor signaling for primary decidua formation. Elife. 2020;9:8.

46. Yuan J, Aikawa S, Deng W, Bartos A, Walz G, Grahammer F, et al. Primary decidual zone formation requires Scribble for pregnancy success in mice. Nat Commun. 2019;10:5425

47. Cha J, Bartos A, Park C, Sun X, Li Y, Cha SW, et al. Appropriate crypt formation in the uterus for embryo homing and implantation requires Wnt5aROR signaling. Cell Rep. 2014;8:382-92.

48. Lee KY, Jeong JW, Wang J, Ma L, Martin JF, Tsai SY, et al. Bmp2 is critical for the murine uterine decidual response. Mol Cell Biol. 2007;27:5468-78.
49. Rogers PA, Murphy CR, Rogers AW, Gannon BJ. Capillary patency and permeability in the endometrium surrounding the implanting rat blastocyst. Int J Microcirc Clin Exp. 1983:2:241-9.

50. Nowotschin S, Hadjantonakis AK. Cellular dynamics in the early mouse embryo: from axis formation to gastrulation. Curr Opin Genet Dev. 2010;20:420-7.

51. Li M, Hu J, Yao L, Gao M. Decreased ANGPTL4 impairs endometrial angiogenesis during peri-implantation period in patients with recurrent implantation failure. J Cell Mol Med. 2020;24:10730-43.

52. Alva JA, Zovein AC, Monvoisin A, Murphy T, Salazar A, Harvey NL, et al. VECadherin-Cre-recombinase transgenic mouse: a tool for lineage analysis and gene deletion in endothelial cells. Dev Dyn. 2006;235:759-67.

53. Munoz-Fernandez R, de la Mata C, Prados A, Perea A, Ruiz-Magana MJ, Llorca T, et al. Human predecidual stromal cells have distinctive characteristics of pericytes: Cell contractility, chemotactic activity, and expression of pericyte markers and angiogenic factors. Placenta. 2018;61:39-47.

54. Lyall F, Bulmer JN, Duffie E, Cousins F, Theriault A, Robson SC. Human trophoblast invasion and spiral artery transformation: the role of PECAM-1 in normal pregnancy, preeclampsia, and fetal growth restriction. Am J Pathol. 2001;158:1713-21.

55. Gellersen B, Wolf A, Kruse M, Schwenke M, Bamberger AM. Human endometrial stromal cell-trophoblast interactions: mutual stimulation of chemotactic migration and promigratory roles of cell surface molecules CD82 and CEACAM1. Biol Reprod. 2013:88:80.

56. Wang Q, Lu J, Zhang S, Wang S, Wang W, Wang B, et al. Wnt6 is essential for stromal cell proliferation during decidualization in mice. Biol Reprod. 2013;88:5.

57. Zenclussen AC, Hammerling GJ. Cellular regulation of the uterine microenvironment that enables embryo implantation. Front Immunol. 2015;6:321

58. Fang X, Ni N, Gao Y, Lydon JP, Ivanov I, Rijnkels M, et al. Transforming growth factor beta signaling and decidual integrity in micedagger. Biol Reprod. 2020;103:1186-98.

59. Lima PD, Zhang J, Dunk C, Lye SJ, Croy BA. Leukocyte driven-decidual angiogenesis in early pregnancy. Cell Mol Immunol. 2014;11:522-37.

\section{Publisher's Note}

Springer Nature remains neutral with regard to jurisdictional claims in published maps and institutional affiliations.

Ready to submit your research? Choose BMC and benefit from

- fast, convenient online submission

- thorough peer review by experienced researchers in your field

- rapid publication on acceptance

- support for research data, including large and complex data types

- gold Open Access which fosters wider collaboration and increased citations

- maximum visibility for your research: over $100 \mathrm{M}$ website views per year

At BMC, research is always in progress.

Learn more biomedcentral.com/submissions 